\title{
CRITICAL EXPONENTS FOR A DOUBLY DEGENERATE PARABOLIC SYSTEM COUPLED VIA NONLINEAR BOUNDARY FLUX
}

\author{
Yongsheng Mi, Chunlai Mu, and Botao Chen
}

\begin{abstract}
The paper deals with the degenerate parabolic system with nonlinear boundary flux. By constructing the self-similar supersolution and subsolution, we obtain the critical global existence curve. The critical Fujita curve is conjectured with the aid of some new results.
\end{abstract}

\section{Introduction}

In this paper, we consider the following doubly degenerate parabolic equations

$$
u_{t}=\left(\left|u_{x}\right|^{p_{1}}\left(u^{m_{1}}\right)_{x}\right)_{x}, \quad v_{t}=\left(\left|v_{x}\right|^{p_{2}}\left(v^{m_{2}}\right)_{x}\right)_{x}, \quad x>0,0<t<T
$$

coupled via nonlinear boundary flux

$$
\begin{cases}-\left|u_{x}\right|^{p_{1}}\left(u^{m_{1}}\right)_{x}(0, t)=v^{q_{1}}(0, t), & 0<t<T, \\ -\left|v_{x}\right|^{p_{2}}\left(v^{m_{2}}\right)_{x}(0, t)=u^{q_{2}}(0, t), & 0<t<T,\end{cases}
$$

and initial data

$$
u(x, 0)=u_{0}(x), \quad v(x, 0)=v_{0}(x), \quad x>0,
$$

where parameters $m_{i} \geq 1, p_{i}>0, q_{i}>0(i=1,2)$, and $u_{0}, v_{0}$ are nonnegative continuous functions with compact support in $\mathbb{R}_{+}$.

Nonlinear parabolic equations (1.1) appear in population dynamics, chemical reactions, heat transfer, and so on, where $u(x, t)$ and $v(x, t)$ represent the densities of two biological populations during a migration, the thickness of two kinds of chemical reactants in a chemical reaction, or the temperatures of two kinds of porous materials during a propagation.

It is well known that the local existence of the weak solution to the problem (1.1)-(1.3), defined in the usual integral way, as well as, a comparison principle can be easily established (see the survey [13] and books $[4,19,31]$ ).

Received November 24, 2009; Revised January 18, 2010.

2010 Mathematics Subject Classification. 35K55, 35K65, 35B40.

Key words and phrases. critical global existence curve, degenerate parabolic systems, critical Fujita curve, nonlinear boundary flux, blow-up. 
The problems on blow-up and global existence conditions, blow-up rates to nonlinear parabolic equations have been intensively studied (see $[1,2,3,5$, $8,11,10,13,16,17,18,21,23,26,30,31,28,29,32,34,35]$ and references therein). In particular, the critical Fujita exponents are very interesting for various nonlinear parabolic equations of mathematical physics (see [3, 15, 18, $26,30,31,28,29,32,34,35]$ and references therein). The concept of critical Fujita exponents was proposed by Fujita in the 1960s during discussion of the heat conduction equation with a nonlinear source (see [6]).

Now we recall some known results. In [8], Galaktionov and Levine studied the single equation case

$$
\begin{cases}u_{t}=\left(u^{m}\right)_{x x}, & x>0,0<t<T, \\ -\left(u^{m}\right)_{x}(0, t)=u^{p}(0, t), & 0<t<T, \\ u(x, 0)=u_{0}(x), & x>0,\end{cases}
$$

and the heat conduction equation with gradient diffusion

$$
\left\{\begin{array}{lc}
u_{t}=\left(\left|u_{x}\right|^{m-1} u_{x}\right)_{x}, & x>0,0<t<T, \\
-\left|u_{x}\right|^{m-1} u_{x}(0, t)=u^{p}(0, t), & 0<t<T, \\
u(x, 0)=u_{0}(x), & x>0,
\end{array}\right.
$$

with $m \geq 1, p>0$ and $u_{0}$ has compact support. They proved that for the problem (1.4) the critical global exponent is $p_{0}=\frac{1}{2}(m+1)$ and the critical Fujita exponent is $p_{c}=m+1$, while for the problem (1.5) the critical global exponent is $p_{0}=\frac{2 m}{m+1}$ and the critical Fujita exponent is $p_{c}=2 m$. The critical global existence exponent and the critical Fujita exponent of (1.5) were also considered in [8] for the special case $m=1$.

Wang and Yin [30], $\mathrm{Li}$ and $\mathrm{Mu}$ [16] studied the following single equation

$$
\begin{cases}u_{t}=\left(\left|\left(u^{m}\right)_{x}\right|^{p-2}\left(u^{m}\right)_{x}\right)_{x}, & x>0,0<t<T, \\ -\left|\left(u^{m}\right)_{x}\right|^{p-2}\left(u^{m}\right)_{x}(0, t)=u^{q}(0, t), & 0<t<T, \\ u(x, 0)=u_{0}(x), & x>0,\end{cases}
$$

where $m>1, p>2, q>0$ and $m>0,1<p<1+\frac{1}{m}, q>0$, respectively, they showed that the critical global existence exponent and critical Fujita exponent are $p_{0}=\frac{(m+1)(p-1)}{p}$ and $p_{c}=(m+1)(p-1)$.

In [28], Wang et al. considered the following problem

$$
\begin{gathered}
u_{t}=u_{x x}, v_{t}=v_{x x}, x>0,0<t<T, \\
-u_{x}(0, t)=v^{p}(0, t),-v_{x}(0, t)=u^{q}(0, t), 0<t<T, \\
u(x, 0)=u_{0}(x), v(x, 0)=v_{0}(x), x>0 .
\end{gathered}
$$

Under some assumptions they established the blow-up estimate near the blowup time.

In [29], Wang et al. considered the following problem

$$
u_{t}=u_{x x}, v_{t}=v_{x x}, x>0,0<t<T,
$$




$$
\begin{gathered}
-u_{x}(0, t)=u^{\alpha} v^{p}(0, t),-v_{x}(0, t)=v^{\beta} u^{q}(0, t), 0<t<T, \\
u(x, 0)=u_{0}(x), v(x, 0)=v_{0}(x), x>0 .
\end{gathered}
$$

The global existence and blow-up conditions for solutions of (1.10)-(1.12) are $p q \leq(1-\beta)(1-\beta)$ and $p q>(1-\beta)(1-\beta)$, respectively. The blow-up rate of the solution $(u, v)$ is $\left(O\left((T-t)^{-\gamma_{1}}\right), O\left((T-t)^{-\gamma_{2}}\right)\right)$ as $t \rightarrow T$ with $\alpha<1, \beta<1$ and $p q \geq(1-\beta)(1-\beta)$, where

$$
\gamma_{1}=\frac{1}{2} \frac{p+1-\beta}{p q-(1-\alpha)(1-\beta)}, \quad \gamma_{2}=\frac{1}{2} \frac{q+1-\alpha}{p q-(1-\alpha)(1-\beta)} .
$$

In [23], Quiros and Rossi considered the degenerate equation

$$
\begin{gathered}
u_{t}=\left(u^{m}\right)_{x x}, v_{t}=\left(v^{n}\right)_{x x}, \quad x>0,0<t<T, \\
-\left(u^{m}\right)_{x}(0, t)=v^{p}(0, t),-\left(v^{n}\right)_{x}(0, t)=u^{q}(0, t), 0<t<T, \\
u(x, 0)=u_{0}(x), v(x, 0)=v_{0}(x), x>0
\end{gathered}
$$

with notation

$$
\begin{array}{llrl}
\alpha_{1} & =\frac{2 p+n+1}{(m+1)(n+1)-4 p q}, & \alpha_{2} & =\frac{2 q+m+1}{(m+1)(n+1)-4 p q}, \\
\beta_{1} & =\frac{p(m-1-2 q)+(n+1) m}{(m+1)(n+1)-4 p q}, & \beta_{2} & =\frac{q(n-1-2 p)+(m+1) n}{(m+1)(n+1)-4 p q} .
\end{array}
$$

They proved that the solutions of (1.13)-(1.15) are global if $p q \leq \frac{1}{4}(m+1)(n+1)$, and may blow up in finite time if $p q>\frac{1}{4}(m+1)(n+1)$. In the case of $p q>\frac{1}{4}(m+1)(n+1)$, if $\alpha_{1}+\beta_{1} \leq 0$, or $\alpha_{2}+\beta_{2} \leq 0$, then every non-negative, non-trivial solutions of (1.13)-(1.15) blow up in finite time: if $\alpha_{1}+\beta_{1}>0$ and $\alpha_{2}+\beta_{2}>0$, then there exist blow-up solutions for large initial and global solutions for small initial data. The critical Fujita exponents to (1.13)-(1.15) are described by $\alpha_{i}+\beta_{i}=0, i=1,2$, while the blow-up rate of the positive solution is $O\left((T-t)^{-\alpha_{1}}\right)$ for component $u$ and $O\left((T-t)^{-\alpha_{2}}\right)$ for $v$ as $t \rightarrow T$.

In [7], Galaktionov and Levine studied the following single equation

$$
\begin{aligned}
& u_{t}=\nabla\left(|\nabla u|^{\sigma} \nabla u^{m}\right)+u^{p}, x \in \mathbb{R}^{N}, t>0, \\
& u(x, 0)=u_{0}(x), \quad x \in \mathbb{R}^{N},
\end{aligned}
$$

where $\sigma>0, \quad m>1, \quad p>1$ and $u_{0}(x)$ is a bounded positive continuous function. They shown that the critical exponent is $p_{c}=m+\sigma+\frac{\sigma+2}{N}$.

Recently, Jiang and Zheng [10] studied the following single equation:

$$
\begin{cases}u_{t}=\left(\left|u_{x}\right|^{\beta}\left(u^{m}\right)_{x}\right)_{x}, & x>0,0<t<T, \\ -\left|u_{x}\right|^{\beta}\left(u^{m}\right)_{x}(0, t)=u^{p}(0, t), & 0<t<T, \\ u(x, 0)=u_{0}(x), & x>0,\end{cases}
$$

where $m \geq 1, p>0, \beta>0$. They obtained the critical global existence exponent $p_{0}=\frac{2 \beta+m+1}{\beta+2}$ and the critical Fujita exponent $p_{c}=2 \beta+m+1$. These results are the extensions of those of Galaktionov and Levine [8]. 
Motivated by the above mentioned works, the aim of this paper is twofold. On the one hand, we construct the self-similar supersolutions and subsolutions to obtain the critical global existence curve of the system (1.1)-(1.3). On the other hand, the critical curve of Fujita type is conjectured with the aid of some new results. The fact that we are dealing with a system instead of a single equation forces us to develop some new techniques.

To state our results, we need to introduce the following numbers. Let

$$
\begin{aligned}
& k_{1}=\frac{\left(p_{2}+1\right)\left(p_{1}+2\right) q_{1}+\left(p_{1}+1\right)\left(2 q_{2}+m_{2}+1\right)}{(p+2)(p+2) q_{1} q_{2}-\left(2 q_{1}+m_{1}+1\right)\left(2 q_{2}+m_{2}+1\right)}, \\
& k_{2}=\frac{\left(p_{1}+1\right)\left(p_{2}+2\right) q_{2}+\left(p_{2}+1\right)\left(2 q_{1}+m_{1}+1\right)}{(p+2)(p+2) q_{1} q_{2}-\left(2 q_{1}+m_{1}+1\right)\left(2 q_{2}+m_{2}+1\right)}, \\
& l_{1}=\frac{k_{2} q_{1}-p_{1} k_{1}-m_{1} k_{1}}{p_{1}+1}, \quad l_{2}=\frac{k_{1} q_{2}-p_{2} k_{2}-m_{2} k_{2}}{p_{2}+1},
\end{aligned}
$$

if $q_{1} q_{2} \neq \frac{\left(2 p_{1}+m_{1}+1\right)\left(2 p_{2}+m_{2}+1\right)}{\left(p_{1}+1\right)\left(p_{2}+2\right)}$. The values $k_{1}, k_{2}, l_{1}, l_{2}$ are the exponents of self-similar solutions to problem (1.1)-(1.3).

Our main results in this paper are stated as follows.

Theorem 1.1. (1) If $q_{1} q_{2} \leq \frac{\left(2 p_{1}+m_{1}+1\right)\left(2 p_{2}+m_{2}+1\right)}{\left(p_{1}+2\right)\left(p_{2}+2\right)}$, then every nonnegative solution of the system (1.1)-(1.3) is global in time;

(2) If $q_{1} q_{2}>\frac{\left(2 p_{1}+m_{1}+1\right)\left(2 p_{2}+m_{2}+1\right)}{\left(p_{1}+2\right)\left(p_{2}+2\right)}$, then the system (1.1)-(1.3) has a solution that blows up in a finite time.

Theorem 1.2. Assume $q_{1} q_{2}>\frac{\left(2 p_{1}+m_{1}+1\right)\left(2 p_{2}+m_{2}+1\right)}{\left(p_{1}+2\right)\left(p_{2}+2\right)}$.

(1) If $\max \left\{l_{1}-k_{1}, l_{2}-k_{2}\right\}<0$, then every nonnegative nontrivial solution of the system (1.1)-(1.3) blows up in finite time.

(2) If $\min \left\{l_{1}-k_{1}, l_{2}-k_{2}\right\}>0$, then there exists a global solution to the system (1.1)-(1.3).

Remark 1.1. Theorem 1.1 show that the critical global existence curve of (1.1)(1.3) is $q_{1} q_{2}=\frac{\left(2 p_{1}+m_{1}+1\right)\left(2 p_{2}+m_{2}+1\right)}{\left(p_{1}+1\right)\left(p_{2}+2\right)}$, the restriction $\max \left\{l_{1}-k_{1}, l_{2}-k_{2}\right\}<0$ in the Theorem1.2(2) is rather technical. It comes from the construction of the so-called Zel'dovich-Kompaneetz-Barenblatt profile. We believe that the critical Fujita curve is $\min \left\{l_{1}-k_{1}, l_{2}-k_{2}\right\}=0$.

Remark 1.2. Unfortunately, we cannot obtain the blow-up rates of the nonglobal solution.

The rest of this paper is organized as follows. In Section 2, we consider the critical global existence curve and prove Theorem 1.1. The proof of Theorem 1.2 is shown in Section 3.

\section{Critical global existence curve}

In this section, by constructing self-similar sub- and super-solutions to problem (1.1)-(1.3), we shall prove Theorem 1.1. 
Proof of Theorem 1.1 (1). It is enough to construct global supersolutions with initial data as large as needed. To this purpose, we look for a globally defined in time strict supersolution of self-similar form

$$
\begin{aligned}
& \bar{u}(x, t)=e^{\kappa_{1} t}\left(M+e^{-L_{1} x e^{-\kappa_{2} t}}\right)^{\frac{1}{m_{1}}}, \quad x \geq 0, t \geq 0, \\
& \bar{v}(x, t)=e^{\kappa_{3} t}\left(M+e^{-L_{2} x e^{-\kappa_{4} t}}\right)^{\frac{1}{m_{2}}}, \quad x \geq 0, t \geq 0,
\end{aligned}
$$

where $M=\max \left\{\left\|u_{0}\right\|_{\infty}^{m_{1}}+1,\left\|v_{0}\right\|_{\infty}^{m_{2}}+1\right\}$, the constants $\kappa_{i}>0(i=$ $1,2,3,4)$, and $L_{i}>0(i=1,2)$ are to be determined. Obviously, we have

$$
\bar{u}(x, 0) \geq u_{0}(x), \quad \bar{v}(x, 0) \geq v_{0}(x), \quad x \geq 0 .
$$

After a direct computation, we obtain

$$
\begin{aligned}
& \bar{u}_{t} \geq \kappa_{1} e^{\kappa_{1} t}\left(M+e^{-L_{1} x e^{-\kappa_{2} t}}\right)^{\frac{1}{m_{1}}} \geq \kappa_{1} e^{\kappa_{1} t} M^{\frac{1}{m_{1}}}, \\
&\left(\left|\bar{u}_{x}\right|^{p_{1}}\left(\bar{u}^{m_{1}}\right)_{x}\right. \\
&=-\frac{L_{1}^{p_{1}+1}}{m_{1}^{p_{1}}} e^{p_{1}\left(\kappa_{1}-\kappa_{2}\right) t+\left(m_{1} \kappa_{1}-\kappa_{2}\right) t} e^{-\left(L_{1} x+p_{1} L_{1} x\right) e^{-\kappa_{2} t}}\left(M+e^{-L_{1} x e^{-\kappa_{2} t}}\right)^{p_{1}\left(\frac{1}{m_{1}}-1\right)}, \\
&\left(\left|\bar{u}_{x}\right|^{p_{1}}\left(\bar{u}^{m_{1}}\right)_{x}\right)_{x} \\
& \leq\left(p_{1}+1\right) \frac{L_{1}^{p_{1}+2}}{m_{1}^{p_{1}}} e^{p_{1}\left(\kappa_{1}-\kappa_{2}\right) t+\left(m_{1} \kappa_{1}-2 \kappa_{2}\right) t} M^{p_{1}\left(\frac{1}{m_{1}}-1\right)},
\end{aligned}
$$

and

$$
\bar{v}_{t} \geq \kappa_{3} e^{\kappa_{3} t}\left(M+e^{-L_{2} x e^{-\kappa_{4} t}}\right)^{\frac{1}{m_{2}}} \geq \kappa_{3} e^{\kappa_{3} t} M^{\frac{1}{m_{2}}}
$$

$$
\begin{aligned}
& \left(\left|\bar{v}_{x}\right|^{p_{2}}\left(\bar{v}^{m_{2}}\right)_{x}\right. \\
= & -\frac{L_{2}^{p_{2}+1}}{m_{2}^{p_{2}}} e^{p_{2}\left(\kappa_{3}-\kappa_{4}\right) t+\left(m_{2} \kappa_{3}-\kappa_{4}\right) t} e^{-\left(L_{2} x+p_{2} L_{2} x\right) e^{-\kappa_{4} t}}\left(M+e^{-L_{2} x e^{-\kappa_{4} t}}\right)^{p_{2}\left(\frac{1}{m_{2}}-1\right)}, \\
& \left(\left|\bar{v}_{x}\right|^{p_{2}}\left(\bar{v}^{m_{2}}\right)_{x}\right)_{x}
\end{aligned}
$$$$
\leq\left(p_{2}+1\right) \frac{L_{2}^{p_{2}+2}}{m_{2}^{p_{2}}} e^{p_{2}\left(\kappa_{3}-\kappa_{4}\right) t+\left(m_{2} \kappa_{3}-2 \kappa_{4}\right) t} M^{p_{2}\left(\frac{1}{m_{2}}-1\right)}
$$

in $\mathbb{R}_{+} \times \mathbb{R}_{+}$. On the other hand, on the boundary we have

$$
\begin{aligned}
-\left|u_{x}\right|^{p_{1}}\left(u^{m_{1}}\right)_{x}(0, t) & =\frac{L_{1}^{p_{1}+1}}{m_{1}^{p_{1}}} e^{p_{1}\left(\kappa_{1}-\kappa_{2}\right) t+\left(m_{1} \kappa_{1}-\kappa_{2}\right) t}(M+1)^{p_{1}\left(\frac{1}{m_{1}}-1\right)}, \\
v^{q_{1}}(0, t) & =e^{q_{1} \kappa_{3} t}(M+1)^{\frac{q_{1}}{m_{2}}} \\
-\left|v_{x}\right|^{p_{2}}\left(v^{m_{2}}\right)_{x}(0, t) & =\frac{L_{2}^{p_{2}+1}}{m_{2}^{p_{2}}} e^{p_{2}\left(\kappa_{3}-\kappa_{4}\right) t+\left(m_{2} \kappa_{3}-\kappa_{4}\right) t}(M+1)^{p_{2}\left(\frac{1}{m_{2}}-1\right)} \\
u^{q_{2}}(0, t) & =e^{q_{2} \kappa_{1} t}(M+1)^{\frac{q_{2}}{m_{1}}}
\end{aligned}
$$


Therefore, we can see that $(\bar{u}, \bar{v})$ is a supersolution of problem (1.1)-(1.3) provided that

$$
\begin{aligned}
& \kappa_{1} e^{\kappa_{1} t} M^{\frac{1}{m_{1}}} \geq\left(p_{1}+1\right) \frac{L_{1}^{p_{1}+2}}{m_{1}^{p_{1}}} e^{p_{1}\left(\kappa_{1}-\kappa_{2}\right) t+\left(m_{1} \kappa_{1}-2 \kappa_{2}\right) t} M^{p_{1}\left(\frac{1}{m_{1}}-1\right)}, \\
& \kappa_{3} e^{\kappa_{3} t} M^{\frac{1}{m_{2}}} \geq\left(p_{2}+1\right) \frac{L_{2}^{p_{2}+2}}{m_{2}^{p_{2}}} e^{p_{2}\left(\kappa_{3}-\kappa_{4}\right) t+\left(m_{2} \kappa_{3}-2 \kappa_{4}\right) t} M^{p_{2}\left(\frac{1}{m_{2}}-1\right)},
\end{aligned}
$$

and

$$
\begin{aligned}
& \frac{L_{1}^{p_{1}+1}}{m_{1}^{p_{1}}} e^{p_{1}\left(\kappa_{1}-\kappa_{2}\right) t+\left(m_{1} \kappa_{1}-\kappa_{2}\right) t}(M+1)^{p_{1}\left(\frac{1}{m_{1}}-1\right)} \geq e^{q_{1} \kappa_{3} t}(M+1)^{\frac{q_{1}}{m_{2}}} \\
& \frac{L_{2}^{p_{2}+1}}{m_{2}^{p_{2}}} e^{p_{2}\left(\kappa_{3}-\kappa_{4}\right) t+\left(m_{2} \kappa_{3}-\kappa_{4}\right) t}(M+1)^{p_{2}\left(\frac{1}{m_{2}}-1\right)} \geq e^{q_{2} \kappa_{1} t}(M+1)^{\frac{q_{2}}{m_{1}}}
\end{aligned}
$$

In order to verify the above inequalities, we only need impose

$$
\begin{aligned}
& \kappa_{1} \geq\left(p_{1}+m_{1}\right) \kappa_{1}-\left(p_{1}+2\right) \kappa_{2}, \kappa_{3} \geq\left(p_{2}+m_{2}\right) \kappa_{3}-\left(p_{2}+2\right) \kappa_{4}, \\
& p_{1}\left(\kappa_{1}-\kappa_{2}\right)+m_{1} \kappa_{1}-\kappa_{2} \geq q_{1} \kappa_{3}, p_{2}\left(\kappa_{3}-\kappa_{4}\right)+m_{2} \kappa_{3}-\kappa_{4} \geq q_{2} \kappa_{1},
\end{aligned}
$$

and

$$
\begin{gathered}
\kappa_{1} M^{\frac{1}{m_{1}}} \geq\left(p_{1}+1\right) \frac{L_{1}^{p_{1}+2}}{m_{1}^{p_{1}}} M^{p_{1}\left(\frac{1}{m_{1}}-1\right)}, \\
\kappa_{3} M^{\frac{1}{m_{2}}} \geq\left(p_{2}+1\right) \frac{L_{2}^{p_{2}+2}}{m_{2}^{p_{2}}} M^{p_{2}\left(\frac{1}{m_{2}}-1\right)}, \\
\frac{L_{1}^{p_{1}+1}}{m_{1}^{p_{1}}}(M+1)^{p_{1}\left(\frac{1}{m_{1}}-1\right)} \geq(M+1)^{\frac{q_{1}}{m_{2}}}, \\
\frac{L_{2}^{p_{2}+1}}{m_{2}^{p_{2}}}(M+1)^{p_{2}\left(\frac{1}{m_{2}}-1\right)} \geq(M+1)^{\frac{q_{2}}{m_{1}}} .
\end{gathered}
$$

Now we show that such choice in (2.1)-(2.4) is valid. Firstly, by taking

$L_{1}=m_{1}^{\frac{p_{1}}{p_{1}+1}}(M+1)^{\frac{q_{1}}{\left(p_{1}+1\right) m_{2}}-\frac{p_{1}-m_{1} p_{1}}{m_{1}\left(p_{1}+1\right)}}, \quad L_{2}=m_{2}^{\frac{p_{2}}{p_{2}+1}}(M+1)^{\frac{q_{2}}{\left(p_{2}+1\right) m_{1}}-\frac{p_{2}-m_{2} p_{2}}{m_{2}\left(p_{2}+1\right)}}$, we see that (2.4) holds.

Secondly, to obtain (2.1), we take

$$
\kappa_{1}=\left(p_{1}+m_{1}\right) \kappa_{1}-\left(p_{1}+2\right) \kappa_{2}, \quad \kappa_{3}=\left(p_{3}+m_{3}\right) \kappa_{3}-\left(p_{3}+2\right) \kappa_{4},
$$

which can also written as

$$
\kappa_{2}=\frac{p_{1}+m_{1}-1}{p_{1}+2} \kappa_{1}, \quad \kappa_{4}=\frac{p_{2}+m_{2}-1}{p_{2}+2} \kappa_{3} .
$$

To obtain the inequalities (2.2), we substitute (2.5) into (2.2) and then only need to confirm

$$
\frac{2 p_{1}+m_{1}+1}{p_{1}+2} \kappa_{1} \geq \frac{q_{1}\left(p_{2}+2\right)}{p_{2}+m_{2}-1} \kappa_{4}, \quad \frac{2 p_{2}+m_{2}+1}{p_{2}+m_{2}-1} \kappa_{4} \geq q_{2} \kappa_{1} .
$$


It follows from the assumption

$$
q_{1} q_{2} \leq \frac{\left(2 p_{1}+m_{1}+1\right)\left(2 p_{2}+m_{2}+1\right)}{\left(p_{1}+2\right)\left(p_{2}+2\right)},
$$

that (2.6) is true for suitable $\kappa_{1}$ and $\kappa_{4}$. Finally, we can further choose $\kappa_{1}$ and $\kappa_{3}$ large enough such that inequalities $(2.3)$ hold.

Therefore, we have proved that $(\bar{u}, \bar{v})$ is a global supersolution of system (1.1)-(1.3). The global existence of solutions to problem (1.1)-(1.3) follows from the comparison principle.

(2) To prove the non-existence of global solutions, we construct a blow-up self-similar subsolution of the system. Construct

$$
\begin{array}{lll}
\underline{u}(x, t)=(T-t)^{-k_{1}} f_{1}(\xi), & \xi=x(T-t)^{-l_{1}}, \\
\underline{v}(x, t)=(T-t)^{-k_{2}} f_{2}(\eta), & & \eta=x(T-t)^{-l_{2}},
\end{array}
$$

where $T$ is a positive constant and $f_{1}, f_{2}$ are two compactly supported functions to be determined.

After some computations, we have

$$
\begin{aligned}
\underline{u}_{t} & =(T-t)^{-\left(k_{1}+1\right)}\left(k_{1} f_{1}(\xi)+l_{1} \xi f_{1}{ }^{\prime}(\xi)\right), \\
\left|\underline{u}_{x}\right|^{p_{1}}\left(\underline{u}^{m_{1}}\right)_{x} & =(T-t)^{-p_{1} k_{1}-p_{1} l_{1}-m_{1} k_{1}-l_{1}}\left|f_{1}{ }^{\prime}\right|^{p_{1}}\left(f_{1}^{m_{1}}\right)^{\prime}(\xi), \\
\left(\left|\underline{u}_{x}\right|^{p_{1}}\left(\underline{u}^{m_{1}}\right)_{x}\right)_{x} & =(T-t)^{-p_{1} k_{1}-p_{1} l_{1}-m_{1} k_{1}-2 l_{1}}\left(\left|f_{1}{ }^{\prime}\right|^{p_{1}}\left(f_{1}^{m_{1}}\right)^{\prime}(\xi)\right)^{\prime}, \\
\underline{v}_{t} & =(T-t)^{-\left(k_{2}+1\right)}\left(k_{2} f_{2}(\eta)+l_{2} \eta f_{2}{ }^{\prime}(\eta)\right), \\
\left|\underline{v}_{x}\right|^{p_{2}}\left(\underline{v}^{m_{2}}\right)_{x} & =(T-t)^{-p_{2} k_{2}-p_{2} l_{2}-m_{2} k_{2}-l_{2}}\left|f_{2}{ }^{\prime}\right|^{p_{2}}\left(f_{2}^{m_{2}}\right)^{\prime}(\eta), \\
\left(\left|\underline{v}_{x}\right|^{p_{2}}\left(\underline{v}^{m_{2}}\right)_{x}\right)_{x} & =(T-t)^{-p_{2} k_{2}-p_{2} l_{2}-m_{2} k_{2}-2 l_{2}}\left(\left|f_{2}{ }^{\prime}\right|^{p_{2}}\left(f_{2}^{m_{2}}\right)^{\prime}(\eta)\right)^{\prime}
\end{aligned}
$$

and

$$
\begin{aligned}
\left|\underline{u}_{x}\right|^{p_{1}}\left(\underline{u}^{m_{1}}\right)_{x}(0, t) & =(T-t)^{-p_{1} k_{1}-p_{1} l_{1}-m_{1} k_{1}-l_{1}}\left|f_{1}{ }^{\prime}\right|^{p_{1}}\left(f_{1}^{m_{1}}\right)^{\prime}(0), \\
\underline{v}^{q_{1}}(0, t) & =(T-t)^{-k_{2} q_{1}} f_{2}^{q_{1}}(0), \\
\left|\underline{v}_{x}\right|^{p_{2}}\left(\underline{v}^{m_{2}}\right)_{x}(0, t) & =(T-t)^{-p_{2} k_{2}-p_{2} l_{2}-m_{2} k_{2}-l_{2}}\left|f_{2}{ }^{\prime}\right|^{p_{2}}\left(f_{2}^{m_{2}}\right)^{\prime}(0), \\
\underline{u}^{q_{2}}(0, t) & =(T-t)^{-k_{1} q_{2}} f_{1}^{q_{2}}(0) .
\end{aligned}
$$

Notice that

$$
\begin{array}{ll}
k_{1}+1=p_{1} k_{1}+p_{1} l_{1}+m_{1} k_{1}+2 l_{1}, & p_{1} k_{1}+p_{1} l_{1}+m_{1} k_{1}+l_{1}=k_{2} q_{1}, \\
k_{2}+1=p_{2} k_{2}+p_{2} l_{2}+m_{2} k_{2}+2 l_{2}, & p_{2} k_{2}+p_{2} l_{2}+m_{2} k_{2}+l_{2}=k_{1} q_{2} .
\end{array}
$$

Thus, $(\underline{u}, \underline{v})$ is subsolution of (1.1)-(1.3) provided that

$$
\begin{gathered}
\left\{\begin{array}{l}
\left(\left|f_{1}{ }^{\prime}\right|_{1}\left(f_{1}^{m_{1}}\right)^{\prime}(\xi)\right)^{\prime} \geq k_{1} f_{1}(\xi)+l_{1} f_{1}^{\prime}(\xi) \xi, \\
\left(\left|f_{2}\right|^{p_{2}}\left(f_{2}^{m_{2}}\right)^{\prime}(\eta)\right)^{\prime} \geq k_{2} f_{2}(\eta)+l_{2} f_{2}^{\prime}(\eta) \eta,
\end{array}\right. \\
\left\{\begin{array}{l}
-\left|f_{1}{ }^{\prime}\right|^{p_{1}}\left(f_{1}^{m_{1}}\right)^{\prime}(0) \leq f_{2}{ }^{q_{1}}(0), \\
-\left|f_{2}{ }^{\prime}\right|^{p_{2}}\left(f_{2}^{m_{2}}\right)^{\prime}(0) \leq f_{1}{ }^{q_{2}}(0) .
\end{array}\right.
\end{gathered}
$$


Set

$$
f_{1}(\xi)=A_{1}\left(a_{1}-\xi\right)_{+}^{\frac{p_{1}+1}{p_{1}+m_{1}-1}}, \quad f_{2}(\eta)=A_{2}\left(a_{2}-\eta\right)_{+}^{\frac{p_{2}+1}{p_{2}+m_{2}-1}}
$$

where $A_{i}, a_{i}(i=1,2)$ are constants to be determined. It is easy to see that

$$
\begin{aligned}
& f_{1}^{\prime}(\xi)=-A_{1} \frac{p_{1}+1}{p_{1}+m_{1}-1}\left(a_{1}-\xi\right)_{+}^{\frac{p_{1}+1}{p_{1}+m_{1}-1}}-1 \\
& \left|f_{1}^{\prime}\right|^{p_{1}}\left(f_{1}^{m_{1}}\right)^{\prime}=-m_{1} A_{1}^{m_{1}+p_{1}}\left(\frac{p_{1}+1}{p_{1}+m_{1}-1}\right)^{p_{1}+1}\left(a_{1}-\xi\right)_{+}^{\frac{p_{1}+1}{p_{1}+m_{1}-1}}, \\
& \left(\left|f_{1}^{\prime}\right|^{p_{1}}\left(f_{1}^{m_{1}}\right)^{\prime}\right)^{\prime}=m_{1} A_{1}^{m_{1}+p_{1}}\left(\frac{p_{1}+1}{p_{1}+m_{1}-1}\right)^{p_{1}+2}\left(a_{1}-\xi\right)_{+}^{\frac{p_{1}+1}{p_{1}+m_{1}-1}-1},
\end{aligned}
$$

and

$$
\begin{aligned}
& f_{2}^{\prime}(\eta)=-A_{2} \frac{p_{2}+1}{p_{2}+m_{2}-1}\left(a_{2}-\eta\right)_{+}^{\frac{p_{2}+1}{p_{2}+m_{2}-1}}-1 \\
& \left|f_{2}^{\prime}\right|^{p_{2}}\left(f_{2}^{m_{2}}\right)^{\prime}=-m_{2} A_{2}^{m_{2}+p_{2}}\left(\frac{p_{2}+1}{p_{2}+m_{2}-1}\right)^{p_{2}+1}\left(a_{2}-\eta\right)_{+}^{\frac{p_{2}+1}{p_{2}+m_{2}-1}}, \\
& \left(\left|f_{2}^{\prime}\right|^{p_{2}}\left(f_{2}^{m_{2}}\right)^{\prime}\right)^{\prime}=m_{2} A_{2}^{m_{2}+p_{2}}\left(\frac{p_{2}+1}{p_{2}+m_{2}-1}\right)^{p_{2}+2}\left(a_{2}-\eta\right)_{+}^{\frac{p_{2}+1}{p_{2}+m_{2}-1}-1} .
\end{aligned}
$$

Substituting (2.11)-(2.17) into (2.9), then inequalities (2.9) are valid provided that

$$
\begin{gathered}
k_{1} A_{1}\left(a_{1}-\xi\right)_{+}^{\frac{p_{1}+1}{p_{1}+m_{1}-1}}-l_{1} \xi A_{1} \frac{p_{1}+1}{p_{1}+m_{1}-1}\left(a_{1}-\xi\right)_{+}^{\frac{p_{1}+1}{p_{1}+m_{1}-1}-1} \\
-m_{1} A_{1}^{m_{1}+p_{1}}\left(\frac{p_{1}+1}{p_{1}+m_{1}-1}\right)^{p_{1}+2}\left(a_{1}-\xi\right)_{+}^{\frac{p_{1}+1}{p_{1}+m_{1}-1}-1} \leq 0
\end{gathered}
$$

and

$$
\begin{gathered}
k_{2} A_{2}\left(a_{2}-\eta\right)_{+}^{\frac{p_{2}+1}{p_{2}+m_{2}-1}}-l_{2} \eta A_{2} \frac{p_{2}+1}{p_{2}+m_{2}-1}\left(a_{2}-\eta\right)_{+}^{\frac{p_{2}+1}{p_{2}+m_{2}-1}-1} \\
-m_{2} A_{2}^{m_{2}+p_{2}}\left(\frac{p_{2}+1}{p_{2}+m_{2}-1}\right)^{p_{2}+2}\left(a_{2}-\eta\right)_{+}^{\frac{p_{2}+1}{p_{2}+m_{2}-1}-1} \leq 0 .
\end{gathered}
$$

To show that the above two inequalities hold, we choose $a_{1}$ and $a_{2}$ with

$$
a_{1}=\omega_{1} A_{1}^{m_{1}+p_{1}-1}, \quad a_{2}=\omega_{2} A_{2}^{m_{2}+p_{2}-1},
$$

where

$$
\begin{aligned}
& \omega_{1}=\frac{m_{1}\left(p_{1}+m_{1}-1\right)}{k_{1}\left(p_{1}+m_{1}-1\right)+\left|l_{1}\right|\left(p_{1}+1\right)}\left(\frac{p_{1}+1}{p_{1}+m_{1}-1}\right)^{p_{1}+2}, \\
& \omega_{2}=\frac{m_{2}\left(p_{2}+m_{2}-1\right)}{k_{2}\left(p_{2}+m_{2}-1\right)+\left|l_{2}\right|\left(p_{2}+1\right)}\left(\frac{p_{2}+1}{p_{2}+m_{2}-1}\right)^{p_{2}+2} .
\end{aligned}
$$

Here, we remark that the assumptions $q_{1} q_{2}>\frac{\left(2 p_{1}+m_{1}+1\right)\left(2 p_{2}+m_{2}+1\right)}{\left(p_{1}+2\right)\left(p_{2}+2\right)}$ imply $k_{1}>0, k_{2}>0$, then the inequalities (2.9) hold. 
On the other hand, the boundary conditions in (2.10) are satisfied if we have

$$
\begin{aligned}
& A_{1}^{m_{1}+p_{1}} \rho_{1} a_{1}^{\frac{p_{1}+1}{p_{1}+m_{1}-1}} \leq A_{2}^{q_{1}} a_{2}^{\frac{q_{1}\left(p_{2}+1\right)}{p_{2}+m_{2}-1}}, \\
& A_{2}^{m_{2}+p_{2}} \rho_{2} a_{2}^{\frac{p_{2}+1}{p_{2}+m_{2}-1}} \leq A_{1}^{q_{2}} a_{1}^{\frac{q_{2}\left(p_{1}+1\right)}{p_{1}+m_{1}-1}}
\end{aligned}
$$

where $\rho_{1}=m_{1}\left(\frac{p_{1}+1}{p_{1}+m_{1}-1}\right)^{p_{1}+1}>0, \quad \rho_{2}=m_{2}\left(\frac{p_{2}+1}{p_{2}+m_{2}-1}\right)^{p_{2}+1}>0$.

According to (2.18), we see that (2.19) and (2.20) hold provided that $A_{1}$ and $A_{2}$ are chosen to satisfy

$$
\begin{aligned}
& A_{1}^{m_{1}+2 p_{1}+1} \rho_{1} \omega_{1}^{\frac{p_{1}+1}{p_{1}+m_{1}-1}} \leq A_{2}^{q_{1}\left(p_{2}+2\right)} \omega_{2}^{\frac{q_{1}\left(p_{2}+1\right)}{p_{2}+m_{2}-1}}, \\
& A_{2}^{m_{2}+2 p_{2}+1} \rho_{2} \omega_{2}^{\frac{p_{2}+1}{p_{2}+m_{2}-1}} \leq A_{1}^{q_{2}\left(p_{1}+2\right)} \omega_{1}^{\frac{q_{2}\left(p_{1}+1\right)}{p_{1}+m_{1}-1}} .
\end{aligned}
$$

The condition $q_{1} q_{2}>\frac{\left(2 p_{1}+m_{1}+1\right)\left(2 p_{2}+m_{2}+1\right)}{\left(p_{1}+2\right)\left(p_{2}+2\right)}$ ensures that we can take $A_{1}$ and $A_{2}$ large enough such that the inequalities (2.21) and (2.22) are valid.

Therefore, if the initial data $u_{0}, v_{0}$ are large enough so that $u_{0}(x) \geq \underline{u}(x, 0)$ and $v_{0}(x) \geq \underline{v}(x, 0)$, then $(\underline{u}, \underline{v})$ is a subsolution to (1.1)-(1.3). By the comparison principle, it implies that the solutions of (1.1)-(1.3) with large initial data blow up in a finite time. The proof is complete.

\section{Critical Fujita curve}

We devote this section to proof of Theorem 1.2. We borrow some ideas from $[8,10]$ to construct suitable auxiliary functions, however, the fact that we are dealing with a system instead of a single equation forces us to develop some new techniques.

Proof of Theorem 1.2 (1). We construct the following well-known self-similar solution (the so-called Zel'dovich-Kompaneetz-Barenblatt profile [8, 13, 24]) to (1.1)-(1.3) in the form

$$
\begin{array}{ll}
u_{B}(x, t)=(\tau+t)^{-\frac{1}{m_{1}+2 p_{1}+1}} h_{1}(\xi), & \xi=x(\tau+t)^{-\frac{1}{m_{1}+2 p_{1}+1}} \\
v_{B}(x, t)=(\tau+t)^{-\frac{1}{m_{2}+2 p_{2}+1}} h_{2}(\eta), & \eta=x(\tau+t)^{-\frac{1}{m_{2}+2 p_{2}+1}}
\end{array}
$$

where $\tau>0$ and

$$
h_{1}(\xi)=C_{1}\left(h_{1}^{\frac{p_{1}+2}{p_{1}+1}}-\xi^{\frac{p_{1}+2}{p_{1}+1}}\right)_{+}^{\frac{p_{1}+1}{p_{1}+m_{1}-1}}, \quad h_{2}(\eta)=C_{2}\left(h_{2}^{\frac{p_{2}+2}{p_{2}+1}}-\eta^{\frac{p_{2}+2}{p_{2}+1}}\right)_{+}^{\frac{p_{2}+1}{p_{2}+m_{2}-1}},
$$

with $h_{1}>0, h_{2}>0$ and

$$
\begin{aligned}
C_{1} & =\left(\frac{1}{m_{1}\left(m_{1}+2 p_{1}+1\right)}\left(\frac{p_{1}+m_{1}-1}{p_{1}+2}\right)^{p_{1}+1}\right)^{\frac{1}{p_{1}+m_{1}-1}}, \\
C_{2} & =\left(\frac{1}{m_{2}\left(m_{2}+2 p_{2}+1\right)}\left(\frac{p_{2}+m_{2}-1}{p_{2}+2}\right)^{p_{2}+1}\right)^{\frac{1}{p_{2}+m_{2}-1}} .
\end{aligned}
$$


It is not difficult to check that

$$
\begin{aligned}
& \left(\left|h_{1}^{\prime}\right|^{p_{1}}\left(h_{1}^{m_{1}}\right)^{\prime}\right)^{\prime}(\xi)+\frac{1}{m_{1}+2 p_{1}+1} \xi h_{1}^{\prime}(\xi)+\frac{1}{m_{1}+2 p_{1}+1} h_{1}(\xi)=0, h_{1}^{\prime}(0)=0, \\
& \left(\left|h_{2}^{\prime}\right|^{p_{2}}\left(h_{2}^{m_{2}}\right)^{\prime}\right)^{\prime}(\eta)+\frac{1}{m_{2}+2 p_{2}+1} \eta h_{2}^{\prime}(\eta)+\frac{1}{m_{2}+2 p_{2}+1} h_{2}(\eta)=0, h_{2}^{\prime}(0)=0 .
\end{aligned}
$$

Since $u(x, t)$ and $v(x, t)$ are nontrivial and nonnegative, we see that $u\left(0, t_{0}\right)>$ 0 and $v\left(0, t_{0}\right)>0$ for some $t_{0}>0$ (compare with a Barenblatt solution of the corresponding equations). Noticing that $u\left(x, t_{0}>0\right), v\left(x, t_{0}\right)>0$ are continuous (see $[9,31]$ ), there exists $\tau>0$ large enough and $h_{1}, h_{2}$ small enough such that

$$
u\left(x, t_{0}\right)>u_{B}\left(x, t_{0}\right), \quad v\left(x, t_{0}\right)>v_{B}\left(x, t_{0}\right) \text { for } x>0 .
$$

A direct calculation shows that $\left(u_{B}(x, t), v_{B}(x, t)\right)$ is a weak subsolution of (1.1)-(1.3) in $(0,+\infty) \times\left(t_{0},+\infty\right)$. By the comparison principle, we obtain that

$$
u(x, t)>u_{B}(x, t), \quad v(x, t)>v_{B}(x, t) \text { for } x>0, t>t_{0} .
$$

Since $\max \left\{l_{1}-k_{1}, l_{2}-k_{2}\right\}<0$, we get $T^{l_{1}} \ll T^{k_{1}}$ and $T^{l_{2}} \ll T^{k_{2}}$ for large $T$. Furthermore, there exists $t * \geq t_{0}$ satisfying

$$
T^{l_{1}} \ll\left(\tau+t^{*}\right)^{\frac{1}{m_{1}+2 p_{1}+1}} \ll T^{k_{1}}, \quad T^{l_{2}} \ll\left(\tau+t^{*}\right)^{\frac{1}{m_{2}+2 p_{2}+1}} \ll T^{k_{2}} .
$$

Let $\underline{u}, \underline{v}$ be the functions defined as in the proof of Theorem 1.1(2). Then for any $x>0$,

$$
\underline{u}(x, 0) \leq u_{B}\left(x, t^{*}\right) \leq u\left(x, t^{*}\right), \quad \underline{v}(x, 0) \leq v_{B}\left(x, t^{*}\right) \leq v\left(x, t^{*}\right) .
$$

It follows from the comparison principle that

$$
\underline{u}(x, t) \leq u\left(x, t+t^{*}\right), \quad \underline{v}(x, t) \leq v\left(x, t+t^{*}\right) \text { for } x>0, t>0 .
$$

As the proof of Theorem 1.1(2), we see that $(\underline{u}, \underline{v})$ blows up in a finite time $T$. Therefore, $\left(u_{1}, u_{2}\right)$ blows up in a finite time which is not larger than $T+t^{*}$. Observing that (3.6) holds for general nontrivial $\left(u_{0}, v_{0}\right)$, and we know that every nonnegative, nontrivial solution of (1.1)-(1.3) blows up in finite time.

(2) Set

$$
\begin{array}{ll}
\bar{u}(x, t)=(\tau+t)^{-k_{1}} F_{1}(\xi), & \xi=x(\tau+t)^{-l_{1}}, \\
\bar{v}(x, t)=(\tau+t)^{-k_{2}} F_{2}(\eta), & \eta=x(\tau+t)^{-l_{2}},
\end{array}
$$

where $k_{i}, l_{i}(i=1,2)$ were defined as before, $T$ is a positive constant and $F_{1}, F_{2}$ are two compactly supported functions to be determined.

After some computations, we have

$$
\begin{aligned}
\bar{u}_{t} & =(\tau+t)^{-\left(k_{1}+1\right)}\left(-k_{1} F_{1}(\xi)-l_{1} \xi F_{1}{ }^{\prime}(\xi)\right), \\
\left|\bar{u}_{x}\right|^{p_{1}}\left(\bar{u}^{m_{1}}\right)_{x} & =(\tau+t)^{-p_{1} k_{1}-p_{1} l_{1}-m_{1} k_{1}-l_{1}}\left|F_{1}{ }^{\prime}\right|^{p_{1}}\left(F_{1}^{m_{1}}\right)^{\prime}(\xi), \\
\left(\left|\bar{u}_{x}\right|^{p_{1}}\left(\bar{u}^{m_{1}}\right)_{x}\right)_{x} & =(\tau+t)^{-p_{1} k_{1}-p_{1} l_{1}-m_{1} k_{1}-2 l_{1}}\left(\left|F_{1}{ }^{\prime}\right|^{p_{1}}\left(F_{1}^{m_{1}}\right)^{\prime}(\xi)\right)^{\prime}, \\
\bar{v}_{t} & =(\tau+t)^{-\left(k_{2}+1\right)}\left(-k_{2} F_{2}(\eta)-l_{2} \eta F_{2}{ }^{\prime}(\eta)\right),
\end{aligned}
$$




$$
\begin{aligned}
\left|\bar{v}_{x}\right|^{p_{2}}\left(\bar{v}^{m_{2}}\right)_{x} & =(\tau+t)^{-p_{2} k_{2}-p_{2} l_{2}-m_{2} k_{2}-l_{2}} \mid F_{2}{ }^{p_{2}}\left(F_{2}^{m_{2}}\right)^{\prime}(\eta), \\
\left(\left|\bar{v}_{x}\right|^{p_{2}}\left(\bar{v}^{m_{2}}\right)_{x}\right)_{x} & =(\tau+t)^{-p_{2} k_{2}-p_{2} l_{2}-m_{2} k_{2}-2 l_{2}}\left(\left|F_{2}{ }^{\prime}\right|^{p_{2}}\left(F_{2}^{m_{2}}\right)^{\prime}(\eta)\right)^{\prime} .
\end{aligned}
$$

and

$$
\begin{aligned}
& \left|\bar{u}_{x}\right|^{p_{1}}\left(\bar{u}^{m_{1}}\right)_{x}(0, t)=(\tau+t)^{-p_{1} k_{1}-p_{1} l_{1}-m_{1} k_{1}-l_{1}}\left|F_{1}{ }^{\prime}\right|^{p_{1}}\left(F_{1}^{m_{1}}\right)^{\prime}(0), \\
& \left|\bar{v}_{x}\right|^{p_{2}}\left(\bar{v}^{m_{2}}\right)_{x}(0, t)=(\tau+t)^{-p_{2} k_{2}-p_{2} l_{2}-m_{2} k_{2}-l_{2}}\left|F_{2}{ }^{\prime}\right|^{p_{2}}\left(F_{2}^{m_{2}}\right)^{\prime}(0), \\
& \bar{v}^{q_{1}}(0, t)=(\tau+t)^{-k_{2} q_{1}} F_{2}{ }^{q_{1}}(0), \quad \bar{u}^{q_{2}}(0, t)=(\tau+t)^{-k_{1} q_{2}} F_{1}{ }^{q_{2}}(0) .
\end{aligned}
$$

Notice that

$$
\begin{array}{ll}
k_{1}+1=p_{1} k_{1}+p_{1} l_{1}+m_{1} k_{1}+2 l_{1}, & p_{1} k_{1}+p_{1} l_{1}+m_{1} k_{1}+l_{1}=k_{2} q_{1}, \\
k_{2}+1=p_{2} k_{2}+p_{2} l_{2}+m_{2} k_{2}+2 l_{2}, & p_{2} k_{2}+p_{2} l_{2}+m_{2} k_{2}+l_{2}=k_{1} q_{2} .
\end{array}
$$

Thus, $(\bar{u}, \bar{v})$ is supsolution of (1.1)-(1.3) provided that

$$
\begin{gathered}
\left\{\begin{array}{l}
\left(\left|F_{1}\right|^{p_{1}}\left(F_{1}^{m_{1}}\right)^{\prime}(\xi)\right)^{\prime}+k_{1} F_{1}(\xi)+l_{1} F_{1}^{\prime}(\xi) \xi \leq 0, \\
\left(\left|F_{2}\right|^{p_{2}}\left(F_{2}^{m_{2}}\right)^{\prime}(\eta)\right)^{\prime}+k_{2} F_{2}(\eta)+l_{2} F_{2}^{\prime}(\eta) \eta \leq 0,
\end{array}\right. \\
\left\{\begin{array}{l}
-\left|F_{1}\right|^{p_{1}}\left(F_{1}^{m_{1}}\right)^{\prime}(0) \geq F_{2}{ }^{q_{1}}(0), \\
-\mid F_{2}{ }^{p_{2}}\left(F_{2}^{m_{2}}\right)^{\prime}(0) \geq F_{1}{ }^{q_{2}}(0),
\end{array}\right.
\end{gathered}
$$

we choose

$$
\begin{aligned}
& F_{1}(\xi)=A_{1} C_{1}\left(\left(a_{1} b_{1}\right)^{\frac{p_{1}+2}{p_{1}+1}}-\left(\xi+a_{1}\right)^{\frac{p_{1}+2}{p_{1}+1}}\right)_{+}^{\frac{p_{1}+1}{p_{1}+m_{1}-1}}=A_{1} h_{1}\left(\xi+a_{1}\right), \\
& F_{2}(\eta)=A_{2} C_{2}\left(\left(a_{2} b_{2}\right)^{\frac{p_{2}+2}{p_{2}+1}}-\left(\eta+a_{2}\right)^{\frac{p_{2}+2}{p_{2}+1}}\right)_{+}^{\frac{p_{2}+1}{p_{2}+m_{2}-1}}=A_{2} h_{2}\left(\eta+a_{2}\right),
\end{aligned}
$$

where $C_{1}$ and $C_{2}$ were defined by (3.4) and (3.5), $h_{1}$ and $h_{2}$ were defined by (3.3), $a_{i}>0, b_{i}>1, A_{i}>0(i=1,2)$. We claim that exist $A_{i}, b_{i}, a_{i}(i=1,2)$ such that the inequalities (3.9) are valid for $F_{1}, F_{2}$ defined by (3.10) and (3.11), then $h_{1}\left(\xi+a_{1}\right)$ and $h_{2}\left(\eta+a_{2}\right)$ satisfy the following equations

$$
\begin{aligned}
& \left(\left|h_{1}^{\prime}\right|^{p_{1}}\left(h_{1}^{m_{1}}\right)^{\prime}\right)^{\prime}=-\frac{1}{m_{1}+p_{1}+1}\left(\xi+a_{1}\right) h_{1}^{\prime}-\frac{1}{m_{1}+p_{1}+1} h_{1}, \\
& \left(\left|h_{2}^{\prime}\right|^{p_{2}}\left(h_{2}^{m_{2}}\right)^{\prime}\right)^{\prime}=-\frac{1}{m_{2}+p_{2}+1}\left(\eta+a_{2}\right) h_{2}^{\prime}-\frac{1}{m_{2}+p_{2}+1} h_{2}
\end{aligned}
$$

and

(3.15) $h_{1}^{\prime}\left(\xi+a_{1}\right)$

$$
=-C_{1} \frac{p_{1}+2}{p_{1}+m_{1}-1}\left(\left(a_{1} b_{1}\right)^{\frac{p_{1}+2}{p_{1}+1}}-\left(\xi+a_{1}\right)^{\frac{p_{1}+2}{p_{1}+1}}\right)_{+}^{\frac{p_{1}+1}{p_{1}+m_{1}-1}-1}\left(\xi+a_{1}\right)^{\frac{1}{p_{1}+1}},
$$

(3.16) $h_{2}^{\prime}\left(\eta+a_{2}\right)$

$$
=-C_{2} \frac{p_{2}+2}{p_{2}+m_{2}-1}\left(\left(a_{2} b_{2}\right)^{\frac{p_{2}+2}{p_{2}+1}}-\left(\eta+a_{2}\right)^{\frac{p_{2}+2}{p_{2}+1}}\right)_{+}^{\frac{p_{2}+1}{p_{2}+m_{2}-1}-1}\left(\eta+a_{2}\right)^{\frac{1}{p_{2}+1}} .
$$


In fact, when $a_{1} \leq \xi+a_{1} \leq b_{1} a_{1}$ and $a_{2} \leq \xi+a_{2} \leq b_{2} a_{2}$, substituting (3.11)(3.16) into (3.9), denote by $y=\xi+a_{1}, z=\eta+a_{2}$, then (3.9) can be transformed into the following inequality with respect $y, z$

$$
\begin{aligned}
& G_{1}(y)=-e_{1} y^{\frac{p_{1}+2}{p_{1}+1}}+e_{2} a_{1} y^{\frac{1}{p_{1}+1}}-e_{3}\left(a_{1} b_{1}\right)^{\frac{p_{1}+2}{p_{1}+1}} \leq 0, \\
& G_{2}(z)=-\theta_{1} y^{\frac{p_{2}+2}{p_{2}+1}}+\theta_{2} a_{2} y^{\frac{1}{p_{2}+1}}-\theta_{3}\left(a_{2} b_{2}\right)^{\frac{p_{2}+2}{p_{2}+1}} \leq 0,
\end{aligned}
$$

where

$$
\begin{aligned}
e_{1} & =\left(k_{1}-\frac{A_{1}^{m_{1}+p_{1}-1}}{m_{1}+p_{1}-1}\right)+\frac{p_{1}+2}{p_{1}+m_{1}-1}\left(l_{1}-\frac{A_{1}^{m_{1}+p_{1}-1}}{m_{1}+p_{1}-1}\right), \\
e_{2} & =\frac{l_{1}\left(p_{1}+2\right)}{p_{1}+m_{1}-1}, \\
e_{3} & =\frac{A_{1}^{m_{1}+p_{1}-1}}{m_{1}+p_{1}-1}-k_{1}, \\
\theta_{1} & =\left(k_{2}-\frac{A_{2}^{m_{2}+p_{2}-1}}{m_{2}+p_{2}-1}\right)-\frac{p_{2}+2}{p_{2}+m_{2}-1}\left(l_{2}-\frac{A_{2}^{m_{2}+p_{2}-1}}{m_{2}+p_{1}-1}\right), \\
\theta_{2} & =\frac{l_{2}\left(p_{2}+2\right)}{p_{2}+m_{2}-1}, \\
\theta_{3} & =\frac{A_{2}^{m_{2}+p_{2}-1}}{m_{2}+p_{2}-1}-k_{2} .
\end{aligned}
$$

Since $\min \left\{l_{1}-k_{1}, l_{2}-k_{2}\right\}>0$, we can choose a suitable constant $A_{1}>0$ such that $l_{1}>\frac{A_{1}^{m_{1}+p_{1}-1}}{m_{1}+p_{1}-1}>k_{1}$ and $\left(k_{1}-\frac{A_{1}^{m_{1}+p_{1}-1}}{m_{1}+p_{1}-1}\right)+\frac{p_{1}+2}{p_{1}+m_{1}-1}\left(l_{1}-\frac{A_{1}^{m_{1}+p_{1}-1}}{m_{1}+p_{1}-1}\right)>$ 0 for such $A_{1}$, it is easy to verify that $e_{i}>0(i=1,2,3)$ and $G_{1}(y)$ is a concave function with respect to $y^{\frac{1}{p_{1}+1}}$, then $G_{1}(y)$ attains its maximum at $z_{*}=\frac{e_{2} a_{1}}{\left(p_{1}+2\right) e_{1}}$. Therefore, $(3.16)$ is valid provided that

$$
G_{1}\left(z_{*}\right)=a_{1}^{\frac{p_{1}+2}{p_{1}+1}}\left\{\frac{p_{1}+1}{p_{1}+2}\left(\frac{1}{e_{1}\left(p_{1}+2\right)}\right)^{\frac{1}{p_{1}+1}} e_{2}^{\frac{p_{1}+2}{p_{1}+1}}-e_{3} b_{1}^{\frac{p_{1}+2}{p_{1}+1}}\right\} \leq 0 .
$$

So, we only need to choose $b_{1}$ sufficiently large such that

$$
b_{1} \geq \max \left\{\left(\frac{\left(p_{1}+1\right) e_{2}}{(p+2) e_{3}}\right)^{\frac{p_{1}+1}{p_{1}+2}}\left(\frac{e_{2}}{(p+2) e_{1}}\right)^{\frac{1}{p_{1}+2}}, 1\right\} \text {. }
$$

Similarly, there exist $A_{2}>0, b_{2}>0$ such that the inequality (3.17) holds. Consequently, we have proved that inequalities (3.9) are true.

Now we consider the boundary condition (3.10), we only need to show that

$$
\begin{aligned}
& \left(A_{1} C_{1}\right)^{m_{1}+p_{1}} m_{1}\left(\frac{p_{2}+2}{p_{1}+m_{1}-1}\right)^{p_{1}+1}\left(b_{1}^{\frac{p_{1}+2}{p_{1}+1}}-1\right)^{\frac{p_{1}+1}{p_{1}+m_{1}-1}} a_{1}^{\frac{2 p_{1}+m_{1}+1}{p_{1}+m_{1}-1}} \\
\geq & \left(A_{2} C_{2}\right)^{q_{1}}\left(b_{2}^{\frac{p_{2}+2}{p_{2}+1}}-1\right)^{\frac{p_{2}+1}{p_{2}+m_{2}-1}} a_{2}^{\frac{\left(p_{2}+2\right) q_{1}}{p_{2}+m_{2}-1}}
\end{aligned}
$$




$$
\begin{aligned}
& \left(A_{2} C_{2}\right)^{m_{2}+p_{2}} m_{2}\left(\frac{p_{2}+2}{p_{2}+m_{2}-1}\right)^{p_{2}+1}\left(b_{2}^{\frac{p_{2}+2}{p_{2}+1}}-1\right)^{\frac{\left(p_{2}+1\right)}{p_{2}+m_{2}-1}} a_{2}^{\frac{2 p_{2}+m_{2}+1}{p_{2}+m_{2}-1}} \\
\geq & \left(A_{1} C_{1}\right)^{q_{2}}\left(b_{1}^{\frac{p_{1}+2}{p_{1}+1}}-1\right)^{\frac{p_{1}+1}{p_{1}+m_{1}-1}} a_{1}^{\frac{\left(p_{1}+2\right) q_{2}}{p_{1}+m_{1}-1}}
\end{aligned}
$$

where $C_{1}$ and $C_{2}$ were defined by (3.4) and (3.5). For above choosed $A_{i}, d_{i}(i=$ $1,2)$, the assumption $q_{1} q_{2}>\frac{\left(2 p_{1}+m_{1}+1\right)\left(2 p_{2}+m_{2}+1\right)}{\left(p_{1}+2\right)\left(p_{2}+2\right)}$ ensures that there exist $a_{2}$ and $a_{1}$ small enough such that the above inequalities hold.

Therefore, it follows from the comparison principle that $(\bar{u}, \bar{v})$ given by $(3.7)$ and (3.8) is a supersolution of the system (1.1)-(1.3) with $\bar{u}(x, 0) \geq u_{0}(x)$, $\bar{v}(x, 0) \geq v_{0}(x)$, which means that the solutions of (1.1)-(1.3) with small initial data have global existence. The proof of Theorem 1.2 is complete.

Acknowledgements. We would like to thank the anonymous referee for his/her careful reading and useful suggestions, which greatly improved the paper. This work was partially supported by NSF of China (10771226), partially supported by Innovative Talent Training Project, the Third Stage of "211 Project", Chongqing University (Project Number S-09110) and partially supported by Foundation Project of China Yangtze Normal University.

\section{References}

[1] G. Astrita and G. Marrucci, Principles of Non-Newtonian Fluid Mechanics, McGrawHill, New York, 1974.

[2] Z. J. Cui, Critical curves of the non-Newtonian polytropic filtration equations coupled with nonlinear boundary conditions, Nonlinear Anal. 68 (2008), no. 10, 3201-3208.

[3] K. Deng and H. A. Levine, The role of critical exponents in blow-up theorems: the sequel, J. Math. Anal. Appl. 243 (2000), no. 1, 85-126.

[4] E. Dibenedetto, Degenerate Parabolic Equations, Springer-Verlag, Berlin, New York, 1993.

[5] R. Ferreira, A. de Pablo, F. Quiros, and J. D. Rossi, The blow-up profile for a fast diffusion equation with a nonlinear boundary condition, Rocky Mountain J. Math. 33 (2003), no. 1, 123-146.

[6] H. Fujita, On the blowing up of solutions of the Cauchy problem for $u_{t}=\Delta u+u^{1+\alpha}$, J. Fac. Sci. Univ. Tokyo Sect. I 13 (1966), 109-124.

[7] V. A. Galaktionov and H. A. Levine, A general approach to critical Fujita exponents in nonlinear parabolic problems, Nonlinear Anal. 34 (1998), no. 7, 1005-1027.

[8] _ On critical Fujita exponents for heat equations with nonlinear flux conditions on the boundary, Israel J. Math. 94 (1996), 125-146.

[9] A. V. Ivanov, Hölder estimates for quasilinear doubly degenerate parabolic equations, J. Soviet Math. 56 (1991), no. 2, 2320-2347.

[10] Z. X. Jiang and S. N. Zheng, Doubly degenerate paralolic equation with nonlinear inner sources or boundary flux, Doctor Thesis, Dalian University of Tcchnology, In China, 2009

[11] C. H. Jin and J. X. Yin, Critical exponents and non-extinction for a fast diffusive polytropic filtration equation with nonlinear boundary sources, Nonlinear Anal. 67 (2007), no. $7,2217-2223$.

[12] A. S. Kalashnikov, A nonlinear equation arising in the theory of nonlinear filtration, Trudy Sem. Petrovsk. No. 4 (1978), 137-146. 
[13] Some problems of the qualitative theory of second-order nonlinear degenerate parabolic equations, Uspekhi Mat. Nauk 42 (1987), no. 2(254), 135-176, 287.

[14] K. A. Lee, A. Petrosyan, and J. L. Vazquez, Large-time geometric properties of solutions of the evolution p-Laplacian equation, J. Differential Equations 229 (2006), no. 2, 389411.

[15] H. A. Levine, The role of critical exponents in blowup theorems, SIAM Rev. 32 (1990), no. 2, 262-288.

[16] Z. P. Li and C. L. Mu, Critical exponents for a fast diffusive polytropic filtration equation with nonlinear boundary flux, J. Math. Anal. Appl. 346 (2008), no. 1, 55-64.

[17] _ Critical curves for fast diffusive non-Newtonian equations coupled via nonlinear boundary flux, J. Math. Anal. Appl. 340 (2008), no. 2, 876-883.

[18] Z. P. Li, C.L. Mu, and Z. J. Cui, Critical curves for a fast diffusive polytropic filtration system coupled via nonlinear boundary flux, Z. Angew. Math. Phys. 60 (2009), no. 2, 284-298.

[19] G. M. Lieberman, Second Order Parabolic Differential Equations, World Scientific Publishing Co., Inc., River Edge, NJ, 1996.

[20] Z. G. Lin, Blowup behaviors for diffusion system coupled through nonlinear boundary conditions in a half space, Sci. China Ser. A 47 (2004), no. 1, 72-82.

[21] Y. S. Mi and C. L. Mu, Critical exponents for a nonlinear degenerate parabolic system coupled via nonlinear boundary flux, submitted.

[22] M. Pedersen and Z. G. Lin, Blow-up analysis for a system of heat equations coupled through a nonlinear boundary condition, Appl. Math. Lett. 14 (2001), no. 2, 171-176.

[23] F. Quirós and J. D. Rossi, Blow-up sets and Fujita type curves for a degenerate parabolic system with nonlinear boundary conditions, Indiana Univ. Math. J. 50 (2001), no. 1, $629-654$.

[24] A. A. Samarskii, V. A. Galaktionov, S. P. Kurdyumov, and A. P. Mikhailov, Blow-up in Quasilinear Parabolic Equations, Walter de Gruyter, Berlin, 1995.

[25] P. Souplet, Blow-up in nonlocal reaction-diffusion equations, SIAM J. Math. Anal. 29 (1998), no. 6, 1301-1334.

[26] J. L. Vazquez, The Porous Medium Equations: Mathematical Theory, Oxford University Press, Oxford, 2007.

[27] M. X. Wang, The blow-up rates for systems of heat equations with nonlinear boundary conditions, Sci. China Ser. A 46 (2003), no. 2, 169-175.

[28] S. Wang, C. H. Xie, and M. X. Wang, Note on critical exponents for a system of heat equations coupled in the boundary conditions, J. Math. Anal. Appl. 218 (1998), no. 1, 313-324.

[29] - The blow-up rate for a system of heat equations completely coupled in the boundary conditions, Nonlinear Anal. 35 (1999), no. 3, Ser. A: Theory Methods, 389398.

[30] Z. J. Wang, J. X. Yin, and C. P. Wang, Critical exponents of the non-Newtonian polytropic filtration equation with nonlinear boundary condition, Appl. Math. Lett. 20 (2007), no. 2, 142-147.

[31] Z. Q. Wu, J. N. Zhao, J. X. Yin, and H. L. Li, Nonlinear Diffusion Equations, World Scientific Publishing Co. Inc. River Edge, NJ, 2001.

[32] Z. Y. Xiang, Q. Chen, and C. L. Mu, Critical curves for degenerate parabolic equations coupled via non-linear boundary flux, Appl. Math. Comput. 189 (2007), no. 1, 549-559.

[33] S. N. Zheng, X. F. Song, and Z. X. Jiang, Critical Fujita exponents for degenerate parabolic equations coupled via nonlinear boundary flux, J. Math. Anal. Appl. 298 (2004), no. 1, 308-324.

[34] J. Zhou and C. L. Mu, The critical curve for a non-Newtonian polytropic filtration system coupled via nonlinear boundary flux, Nonlinear Anal. 68 (2008), no. 1, 1-11. 
[35] _ On the critical Fujita exponent for a degenerate parabolic system coupled via nonlinear boundary flux, Proc. Edinb. Math. Soc. (2) 51 (2008), no. 3, 785-805.

YONGSHENG Mi

College of Mathematics and Statistics

Chongqing University

ChongQing 400044, P. R. China

AND

College of Mathematics and Computer Sciences

YANGTZE Normal University

Fuling 408100, Chongqing, P. R. China

E-mail address: miyongshen@163.com

Chunlai Mu

College of Mathematics and Statistics

CHONGQING UNIVERSITY

Chongling 400044, P. R. China

E-mail address: chunlaimu@yahoo.com.cn

Botao Chen

College of Mathematics and Computer Sciences

YANGTZE Normal University

Fuling 408100, Chongqing, P. R. China

E-mail address: chenbotao2004@126.com 